\title{
Jingfukang induces anti-cancer activity through oxidative stress-mediated DNA damage in circulating human lung cancer cells
}

Zujun Que ${ }^{1 \dagger}$, Zhiyi Zhou ${ }^{2 \dagger}$, Bin Luo ${ }^{2}$, Changsheng Dong ${ }^{1}$, Yi Jiang ${ }^{2}$, Hegen Li² ${ }^{*}$ and Jianhui Tian ${ }^{1,2^{*}}$

\begin{abstract}
Background: Metastasis is the main cause of lung cancer death. As a seed of metastasis, circulating tumor cells are an important target for metastasis intervention. The traditional Chinese medicine, Jinfukang, has been clinically available for the treatment of non-small cell lung cancer (NSCLC). In this study, we investigated the action and underlying mechanisms of Jinfukang against circulating lung tumor cells.

Methods: The cell counting kit-8 (CCK-8), colony formation and cell cycle assays were used to study the cell proliferation ability. Flow cytometry was used to detect the apoptosis and the expression level of ROS and Caspase-3. Comet and TUNEL assays were used to detect DNA damage. DNA damage related pathway protein was detected by western blot.

Results: Jinfukang significantly inhibits the proliferation of CTC-TJH-01 cells by inducing G1 phase arrest and inhibits their colony formation in a dose-dependent manner. Moreover, Jinfukang induces apoptosis in CTC-TJH-01 cells through the ROS-mediated ATM/ATR-p53 pathway and DNA damage.
\end{abstract}

Conclusions: Our findings suggest that Jinfukang may be a potential drug for lung cancer metastasis.

Keywords: Non-small cell lung cancer, Circulating tumor cell, Jinfukang, Apoptosis, Oxidative stress

\section{Background}

Lung cancer is the leading cause of cancer-associated deaths, $85 \%$ of which is non-small cell lung cancer (NSCLC) [1]. Current multidisciplinary treatment for lung cancer can reduce disease recurrence and increase longterm survival of the patients $[2,3]$. However, existing therapies have limited benefits for lung cancer patients at the early stage. Numerous studies have shown that adjuvant chemotherapy, targeted therapy and immunotherapy do not improve the prognosis and survival of patients with early-stage lung cancer and cause serious side effects and complications [4-6]. The main reason is that current

\footnotetext{
*Correspondence: shlaogen@163.com; hawk7150@hotmail.com

${ }^{\dagger}$ Zujun Que and Zhiyi Zhou contributed equally to this work.

2Department of Oncology, Longhua Hospital, Shanghai University of Traditional Chinese Medicine, No. 725, South Wanping Road, Shanghai 200032, China

${ }^{1}$ Oncology Institute of Traditional Chinese Medicine, Shanghai Research Institute of Traditional Chinese Medicine, No. 725, South Wanping Road, Shanghai 200032, China
}

(c) The Author(s). 2019 Open Access This article is distributed under the terms of the Creative Commons Attribution 4.0 International License (http://creativecommons.org/licenses/by/4.0/), which permits unrestricted use, distribution, and reproduction in any medium, provided you give appropriate credit to the original author(s) and the source, provide a link to the Creative Commons license, and indicate if changes were made. The Creative Commons Public Domain Dedication waiver (http://creativecommons.org/publicdomain/zero/1.0/) applies to the data made available in this article, unless otherwise stated. sign of treatment options for primary lung cancer, but for early lung cancer patients, there is a lack of anti-metastatic drugs [7].

Circulating tumor cells (CTCs) represent the primary cause of intractable metastatic disease and are considered essential for metastasis formation. The latest research shows that the number of CTCs is closely related to the metastasis of lung cancer [8]. A guideline for the clinical practice of malignancy issued by the National Comprehensive Cancer Network (NCCN) has incorporated CTCs into the TNM (tumor node metastasis) staging system [9]. In addition, the eighth edition of the cancer staging system developed by the American Joint Committee on Cancer (AJCC) lists CTCs in the peripheral blood as a prognostic factor of breast cancer [10]. Hashimoto $\mathrm{M}$ and his colleagues found that increasing pvCTC count was significantly correlated with postoperative distant metastasis in completely resected NSCLC patients [11]. Therefore, targeting CTCs to develop specific anti- 
metastatic drugs may be the key to improving the clinical efficacy of the early-stage lung cancer. The current research and development of lung cancer drugs mainly focuses on the tissues and cells of primary tumors, which is the main reason for leading to poor clinical efficacy.

Traditional Chinese Medicine (TCM) has been used to treat for treatment various diseases. Jinfukang oral liquid, a Chinese herbal prescription, consists of 12 Chinese herbal medicines (Additional file 1: Table S1) and was approved by the State Food and Drug Administration in 1996 (Z19991043). In clinical practice, Jinfukang is specifically used in the treatment of NSCLC, which has been proved to be capable of preventing the occurrence of metastasis, stabilizing tumor lesions, improving the response rates when combined with chemotherapy, and prolonging the survival period of lung cancer patients $[12,13]$. However, the clinical effects of Jinfukang are certain, but the biological mechanism is unclear.

In this study, we examined the effects of Jinfukang on a circulating human lung cancer tumor cell line and studied the underlying molecular mechanisms involved, which might provide experimental evidence for clinical therapy in lung cancer.

\section{Methods}

\section{Chemicals and reagents}

Jinfukang oral liquid freeze-dried powder was prepared and detected the fingerprint (Additional file 2: Table S2) by Professor $\mathrm{Yu}$ Jin of East China University of Science and Technology (Shanghai, China). A cell counting kit-8 (CCK-8) was obtained from Dojindo. The Annexin V-FITC apoptosis detection kit and propidium iodide (PI) were purchased from BD Pharmingen. 2,7-Dichlorodihydrofluorescein diacetate (DCFH-DA) and the antioxidant NAC were purchased from Sigma. A caspase-3 detection kit was purchased from Biovision. The anti- $\gamma-\mathrm{H} 2 \mathrm{AX}$, anti-p-ATM, anti-p-ATR, antiPARP1, goat anti-mouse IgG-HRP and donkey anti-rabbit IgG-HRP antibodies were purchased from Cell Signaling Technology. The anti-p53, anti-p21, anti-CDK4, anti-Cyclin $\mathrm{D}$, anti-Cyclin E, anti-Fas, anti-Survivin, and anti- $\beta$-actin antibodies were purchased from Proteintech.

\section{Cell culture}

The circulating human lung cancer cell line CTC-TJH01 was isolated from the peripheral blood of patients with stage II lung cancer [14]. The cells were cultured in F12K medium (Gibco, USA) with 10\% fetal bovine serum (Biological Industries, Israel) and incubated at $37^{\circ} \mathrm{C}$ in a humidified air containing $5 \% \mathrm{CO}_{2}$.

\section{Cell viability assay}

The cells were seeded into 96-well plates at a density of $3 \times 10^{3}$ per well and allowed to grow overnight. Jinfukang was dissolved in DMSO and diluted with F12K medium to the final concentrations of $125,250,500$, and $1000 \mu \mathrm{g} /$ $\mathrm{mL}$. The tumor cells were incubated with Jinfukang for 24, 48 and $72 \mathrm{~h}$ before the CCK-8 assay (Dojindo, Japan). Absorbance values were measured at $450 \mathrm{~nm}$ and survival curves were plotted using GraphPad software.

\section{Colony forming assay}

The CTC-TJH-01 cells were seeded in 6-well plates (300 cells/well), allowed to attach overnight, and then treated with Jinfukang ( 350 or $700 \mu \mathrm{g} / \mathrm{mL}$ ) for 10 days. The cells were fixation with $4 \%$ polyoxymethylene, washed thrice with PBS, and then stained with Giemsa for $30 \mathrm{~min}$. The cells were then scanned with an HP scanner, and each well was counted.

\section{Cell cycle analysis and detection of caspase-3 activation}

For the cell cycle analysis, the cells were stained with PI and analyzed by a FACSVerse ${ }^{\mathrm{in}}$ flow cytometer (BD Biosciences, CA) as described previously [15]. Caspase-3 activation was determined using a CaspGLOW ${ }^{m}$ fluorescein active caspase3 staining kit (Biovision, Mountain View, CA).

\section{Cell apoptosis analysis}

The cells were treated with Jinfukang (350 or $700 \mu \mathrm{g} /$ $\mathrm{mL}$ ) for $48 \mathrm{~h}$, and then collect the cells, washed twice with ice-cold PBS, and evaluated for apoptosis by double stains with FITC-conjugated annexin V and PI in binding buffer for 30 min using a FACSVerse flow cytometer.

\section{Measurement of ROS generation}

Cellular ROS contents were measured by flow cytometry as described previously [15]. Briefly, the cells were treated with Jinfukang ( 350 or $700 \mu \mathrm{g} / \mathrm{mL}$ ) for $48 \mathrm{~h}$, and stained with $10 \mu \mathrm{M}$ DCFH-DA (Sigma, USA) at $37^{\circ} \mathrm{C}$ for $30 \mathrm{~min}$. The cells were collected and the fluorescence was analyzed using a FACSVerse flow cytometer.

\section{TUNEL assay}

The cells were treated with Jinfukang (350 or $700 \mu \mathrm{g} /$ $\mathrm{mL}$ ) for $48 \mathrm{~h}$, and then examined for apoptosis using the in situ cell death detection kit (Roche, China) according to the manufacturer's instruction manual.

\section{Immunofluorescence staining}

The cells were treated with Jinfukang (350 or $700 \mu \mathrm{g} / \mathrm{mL}$ ) for $48 \mathrm{~h}$, fixed with $4 \%$ paraformaldehyde for $30 \mathrm{~min}$, incubated with $0.3 \%$ Triton X-100 for $10 \mathrm{~min}$, and blocked with $0.1 \%$ BSA in PBS for $1 \mathrm{~h}$. The primary anti- $\gamma-\mathrm{H} 2 \mathrm{AX}$ antibody (1:200, CST, USA) was added and incubated overnight at $4{ }^{\circ} \mathrm{C}$, and the cells were incubated for $1 \mathrm{~h}$ with the appropriate secondary antibody. The slides were incubated with $0.5 \mathrm{mg} / \mathrm{ml}$ DAPI (Sigma, USA) for $5 \mathrm{~min}$. All images were 
observed under a confocal microscope equipped with a digital camera (Leica, Germany).

\section{Comet assay}

The Comet assay was performed as previously described [16]. The DNA damage of CTC-TJH-01 cells was detected by single cell gel electrophoresis assay. For analysis of the comet images, the extent of DNA damage was estimated by fluorescence microscopy using the semiquantitative method of visual scoring.

\section{Western blot analysis}

Western blotting was conducted as described previously [17]. In brief, the cell lysates and proteins were extracted. $40 \mu \mathrm{g}$ of the proteins was used to detect. The immunoreactive bands were visualized by using an ECL kit (Bio-Rad, Hercules, CA). The density of the immunoreactive bands was analyzed using ImageJ computer software (National Institutes of Health, MD).

\section{Statistical analysis}

All experiments were assayed in triplicate $(n=3)$. The data are expressed as the mean \pm SEM. All statistical analyses were performed using GraphPad Prism 6.0 (GraphPad, SanDiego, CA). The differences in the measured variables between the experimental and control groups were assessed by the Student's test. $P<0.05$ was defined as statistically significant.

\section{Results}

Jinfukang inhibits proliferation of CTC-TJH-01 cells

The cytotoxicity of Jinfukang to CTC-TJH-01 cells was measured by the CCK-8 assay. We found that Jinfukang decreased the cell viability of the CTC-TJH-01 cells in a time- and dose-dependent manner (Fig. 1a). The $\mathrm{IC}_{50}$ of Jinfukang at $48 \mathrm{~h}$ was $728.8 \pm 4.4 \mu \mathrm{g} / \mathrm{mL}$. In addition, Jinfukang also can inhibited the number of clones of the CTC-TJH-01 cells in a dose-dependent manner (Fig. 1b), and arresting the CTC-TJH-01 cells in the G1 phase (Fig. 1c). These data suggested that Jinfukang exhibits potent cytotoxicity against the CTC-TJH-01 cells.

\section{Jinfukang induces apoptosis in CTC-TJH-01 cells}

We used annexin V/PI staining assay to detect the apoptosis of CTC-TJH-01 cells. It was found that Jinfukang can significantly induce apoptosis in CTC-TJH-01 cells. (Fig. 2a). In addition, we also determined the levels of caspase- 3 and ROS in CTC-TJH-01 cells. The results show that Jinfukang can increase the levels of caspase-3 and ROS (Fig. 2c, e). The above results suggest that Jinfukang-induced apoptosis of CTC-TJH-01 cells may be through ROS pathway activation.

\section{Jinfukang can induce CTC-TJH-01 cells apoptosis through ROS pathway}

To further clarify the mechanism of Jinfukang inducing apoptosis of CTC-TJH-01 cells, the antioxidant $\mathrm{N}$-acetyl cysteine (NAC, $3 \mathrm{mM}$ ) was used in the treatment. It was found that Jinfukang-mediated ROS induction was inhibited (Fig. 2d). Furthermore, we also found that
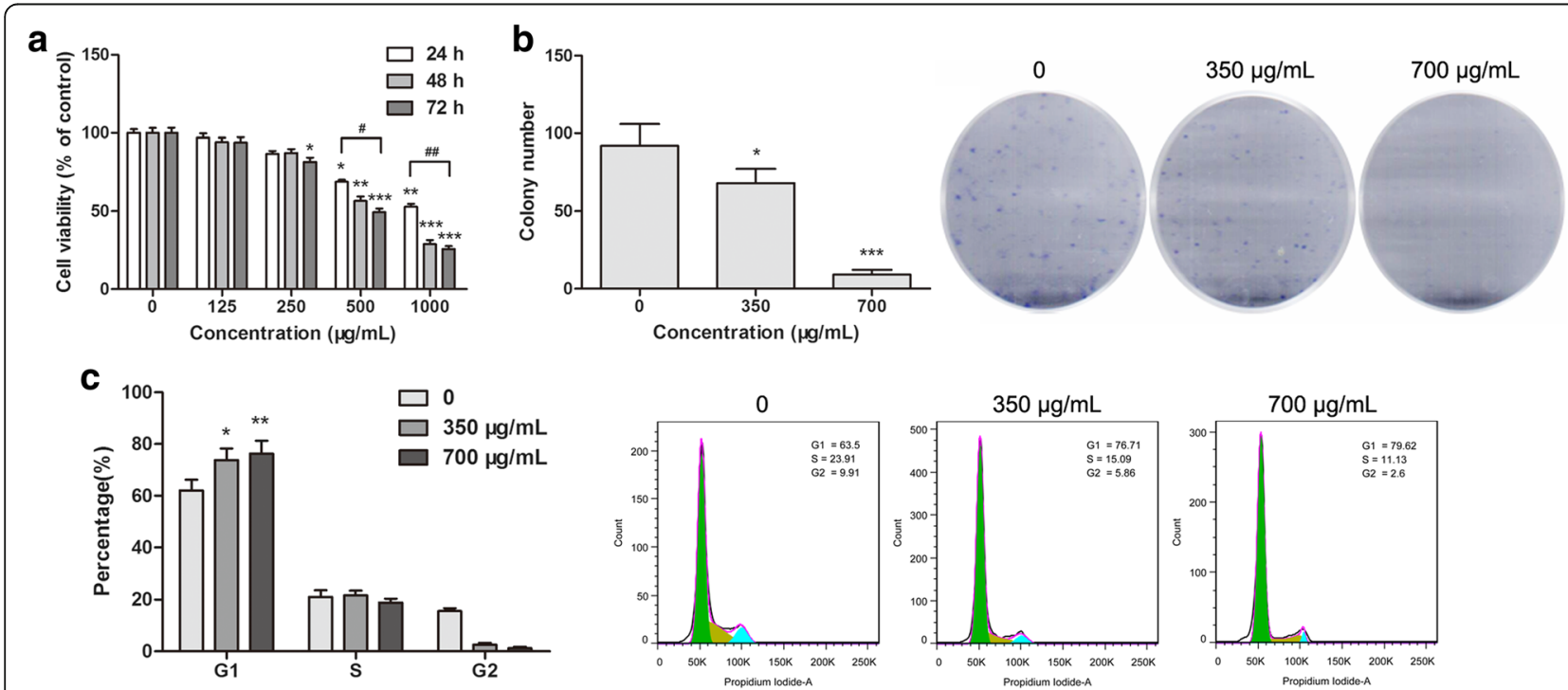

Fig. 1 Jinfukang inhibits CTC-TJH-01 cell growth. a CTC-TJH-01 cells were incubated with Jinfukang (0, 125, 250, 500, and 1000 $\mu \mathrm{g} / \mathrm{mL})$ for $24 \mathrm{~h}$, $48 \mathrm{~h}$ and $72 \mathrm{~h}$. The CCK-8 assay was performed to determine the cytotoxic effect of Jinfukang. $\mathbf{b}$ Representative images of the colony formation assay. c CTC-TJH-01 cells were treated with Jinfukang $(0,350$, and $700 \mu \mathrm{g} / \mathrm{mL})$ for $48 \mathrm{~h}$. Flow cytometry was performed to determine the cell cycle. Each bar represents the means \pm SD of three separate experiments. ${ }^{*} P<0.05$; ${ }^{* *} P<0.01$; ${ }^{* *} P<0.001$ 


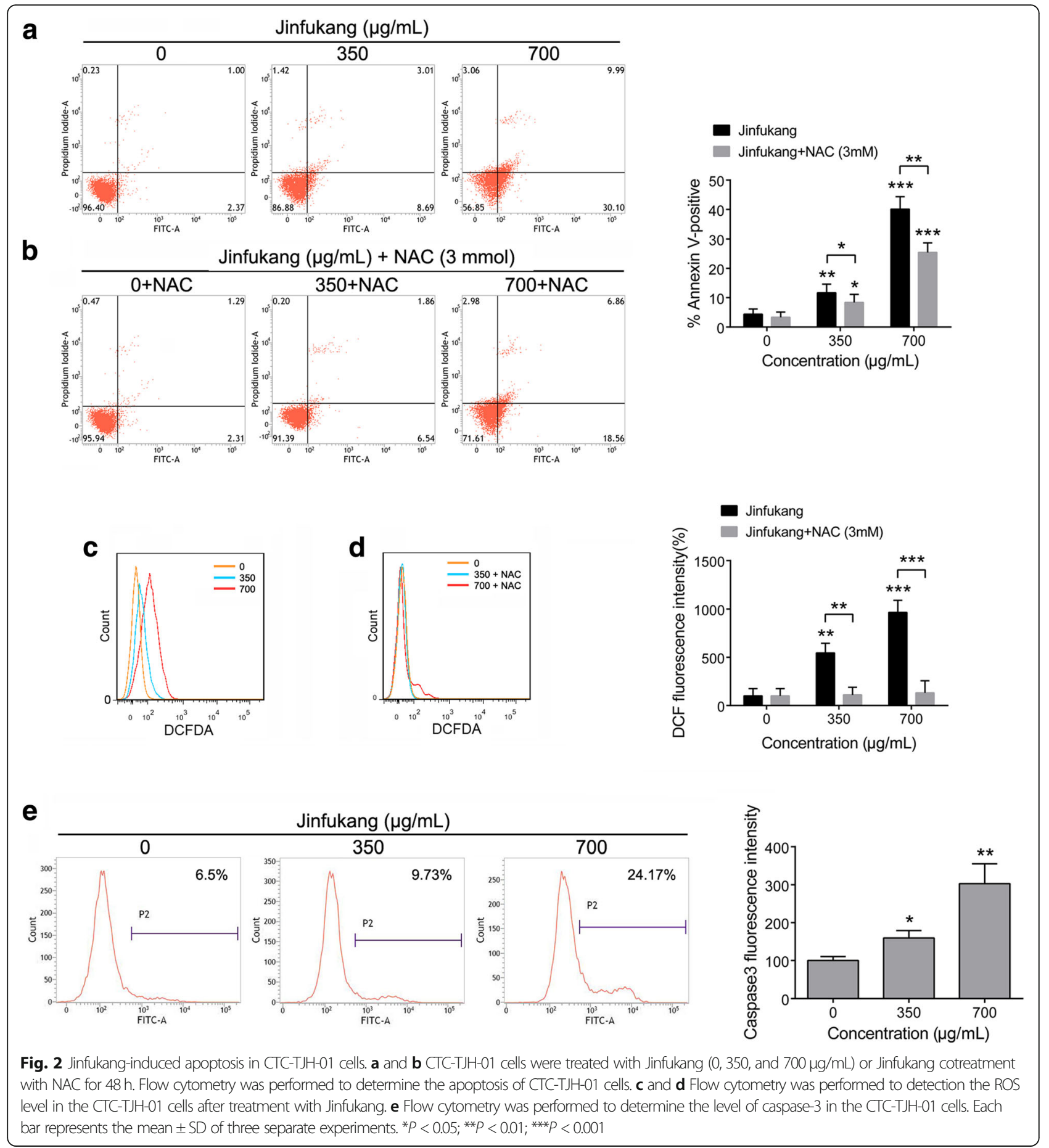

NAC attenuated CTC-TJH-01 cells apoptosis (Fig. 2b). These results proved that Jinfukang-induced growth inhibition and apoptosis of CTC-TJH-01 cells through ROS pathway activation.

\section{Jinfukang can induce DNA damage in CTC-TJH-01 cells} Then, we detected the DNA integrity of CTC-TJH-01 cells. TUNEL detection results show that the DNA damage was increased in the CTC-TJH-01 cells after treatment with Jinfukang (Fig. 3a). Furthermore, we also found that Jinfukang significantly upregulated the expression of $\gamma-\mathrm{H} 2 \mathrm{AX}$ protein in the nucleus of CTCTJH-01 cells (Fig. 3b). These results suggested that Jinfukang may promote the apoptosis of CTC-TJH-01 cells by inducing DNA damage. Hence, the comet assay was also used to confirm that Jinfukang can induce DNA 


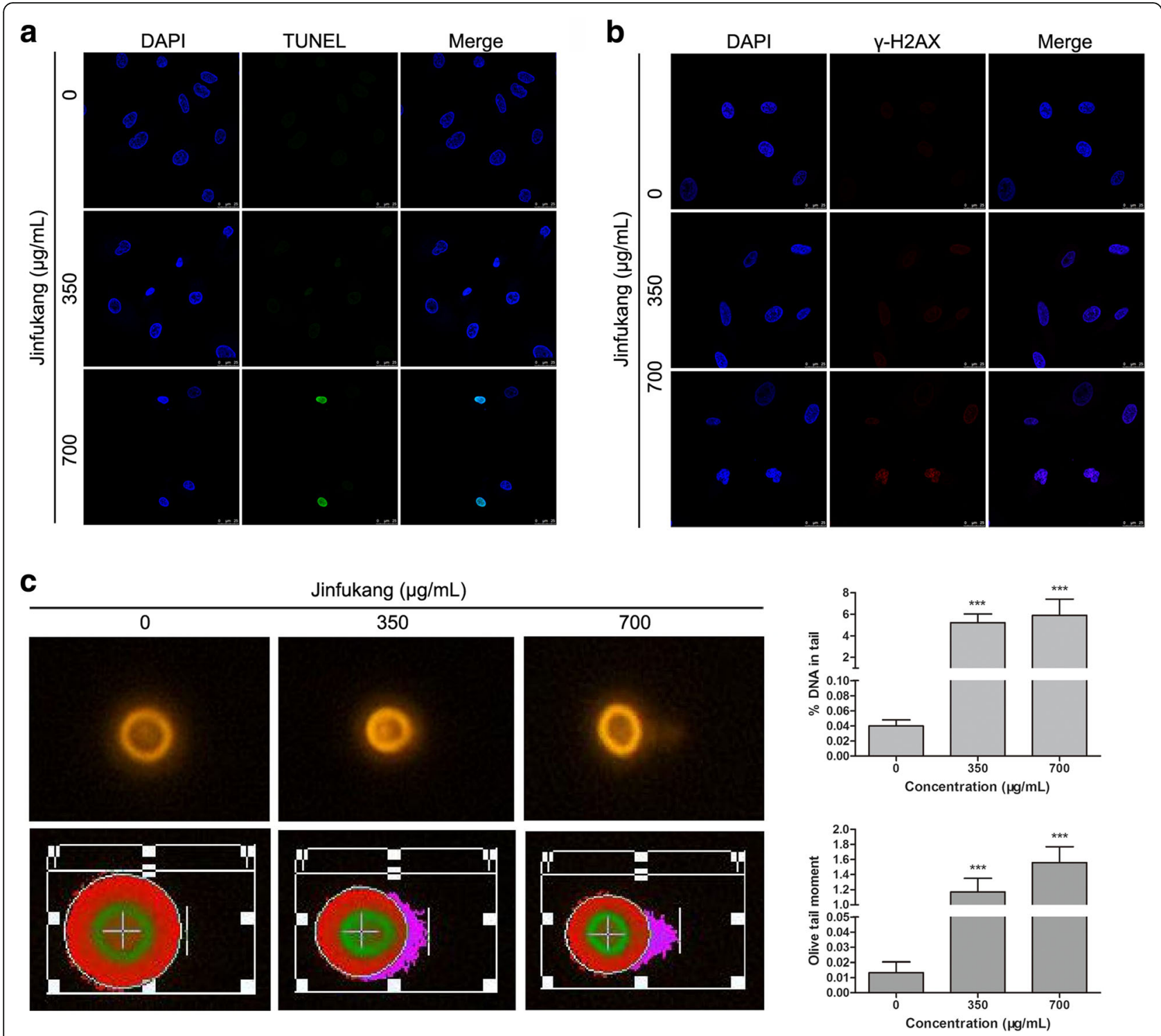

Fig. 3 Jinfukang-induced DNA damage in CTC-TJH-01 cells. a and b CTC-TJH-01 cells were treated with Jinfukang (0, 350, and 700 $\mu \mathrm{g} / \mathrm{mL})$ for 48 h. Immunofluorescence was used to detect DNA damage and $\mathrm{y}-\mathrm{H} 2 \mathrm{AX}$ expression in the CTC-TJH-01 cells. c Detection of nuclear damage in the CTC-TJH-01 cells by comet electrophoresis. Each bar represents the mean \pm SD of three separate experiments. ${ }^{*} P<0.05$; ${ }^{* *} P<0.01$; ${ }^{* * *} P<0.001$

damage in CTC-TJH-01 cells. These results indicate that Jinfukang induce the apoptosis of CTC-TJH-01 cells through ROS-mediated oxidative DNA damage.

\section{Jinfukang induces the apoptosis of CTC-TJH-01 cells through the ROS-mediated ATM/ATR-p53 pathway}

To further reveal the mechanism of Jinfukang inducing CTC-TJH-01 apoptosis, we studied the ROS-mediated DNA damage-related ATM/ATR-p53 pathways. It was found that the expression of phospho-ATM, phosphoATR, PARP1, p53, p21 and Fas proteins was significantly upregulated when the CTC-TJH-01 cells were treated with Jinfukang, and the expression of surviving protein was down-regulated (Fig. 4). In addition, Jinfukang inhibited the expression of the CDK2, CDK4, CDK6, cyclin D1 and cyclin E1 proteins in the CTC-TJH-01 cells (Fig. 4). Taken together, these results indicated that Jinfukang can induce DNA damage in CTC-TJH-01 cells through the ROS-mediated ATM/ATR-p53 signaling pathway, and the proliferation of the CTC-TJH-01 cells is arrested in the G1 phase through the p53-p21 signaling pathway (Fig. 5).

\section{Discussion}

It is well accepted that metastasis is the main cause of lung cancer death, and prevention of postoperative metastasis is an urgent need for current lung cancer treatment [18]. However, metastasis remains the most poorly 

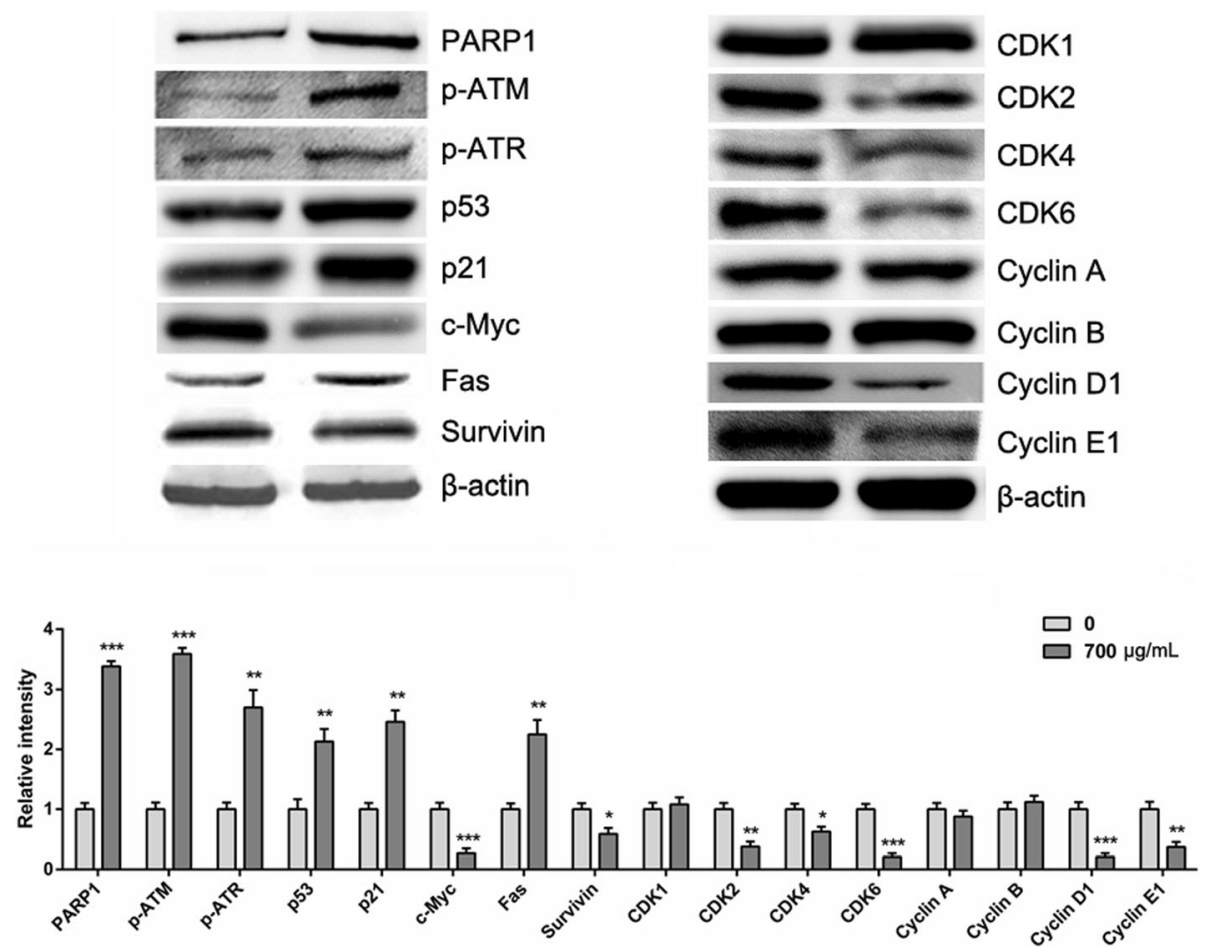

Fig. 4 Apoptosis related protein expression was detected by western blot analysis after treatment of the CTC-TJH-01 cells with Jinfukang (0, 350, $700 \mathrm{\mu g} / \mathrm{mL}$ ) for $48 \mathrm{~h}$. $\beta$-actin was used as an internal standard

understood component of cancer pathogenesis, and most existing drugs inhibit only cancer cell proliferation. There are two complementary anti-metastasis strategies, the prevention of cancer cell dissemination and the suppression of metastases already in existence [7]. The theory of "seed and soil" suggests that circulating tumor cells may be the seeds disseminated by the cancer cells. Recent studies have shown that the number of CTCs is associated with the metastasis and survival of patients with lung cancer [8]. Our previous study found that CTC-TJH-01 cells are in the intermediate stage of EMT transformation, with strong drug resistance, stem cell phenotype and immune escape characteristics. In vivo studies have shown that CTC-TJH-01 cells have tumorigenicity and lung metastasis ability [19]. Therefore, targeting CTCs may be the key to preventing metastasis and improving the survival of patients with lung cancer.

Chinese herbal medicine has been increasingly used for the treatment of cancer. The TCM formula, Jingfukang, has been used for more than 20 years to treat lung cancer in clinical practice. Clinical results have confirmed that Jinfukang can prevent the recurrence and metastasis of lung cancer and prolong the survival of patients [20]. However, the cellular and molecular mechanisms remain unclear. Previous studies have demonstrated that Jingfukang can promote DDP-induced apoptosis in lung cancer cells [21, 22]. In addition, other studies have also found that
Jinfukang can inhibit lymphatic endothelial cell formation and migration and inhibit the lung tumor mass via the suppression of bone marrow-derived mesenchymal stem cell transformation and lung tumor lymphangiogenesis [23, 24].

Based on the above results, we speculate that Jinfukang may inhibit the recurrence and metastasis of lung cancer by interfering with CTCs in the peripheral blood of patients with lung cancer. Therefore, we studied the intervention effect of Jinfukang on the CTC-TJH-01 cells. The results showed that Jinfukang significantly inhibited the proliferation of the CTC-TJH-01 cells, arrested their proliferation in the G1 phase, and inhibited their monoclonal-forming ability. We found that Jinfukang triggered G1 phase arrest in CTC-TJH-01 cells by up-regulating the expression of p53 and p21 levels and down-regulating the expression levels of CDK2, CDK4, CDK6, Cyclin D1, and Cyclin E1. These results are consistent with previous reports showing that p21 can induce G1 phase arrest through suppress Cyclin D1/CDK4 complex [25]. Further study found that Jinfukang significantly induced apoptosis in CTC-TJH-01 cells. These findings are in agreement with previous reports in that Jinfukang can induce apoptosis and cell cycle progression in lung adenocarcinoma cancer cells $[21,22]$.

The present study demonstrates that Jinfukang can induce the apoptosis of lung cancer cells by activating the expression of AIFM2 [21]. In addition, Jinfukang can 


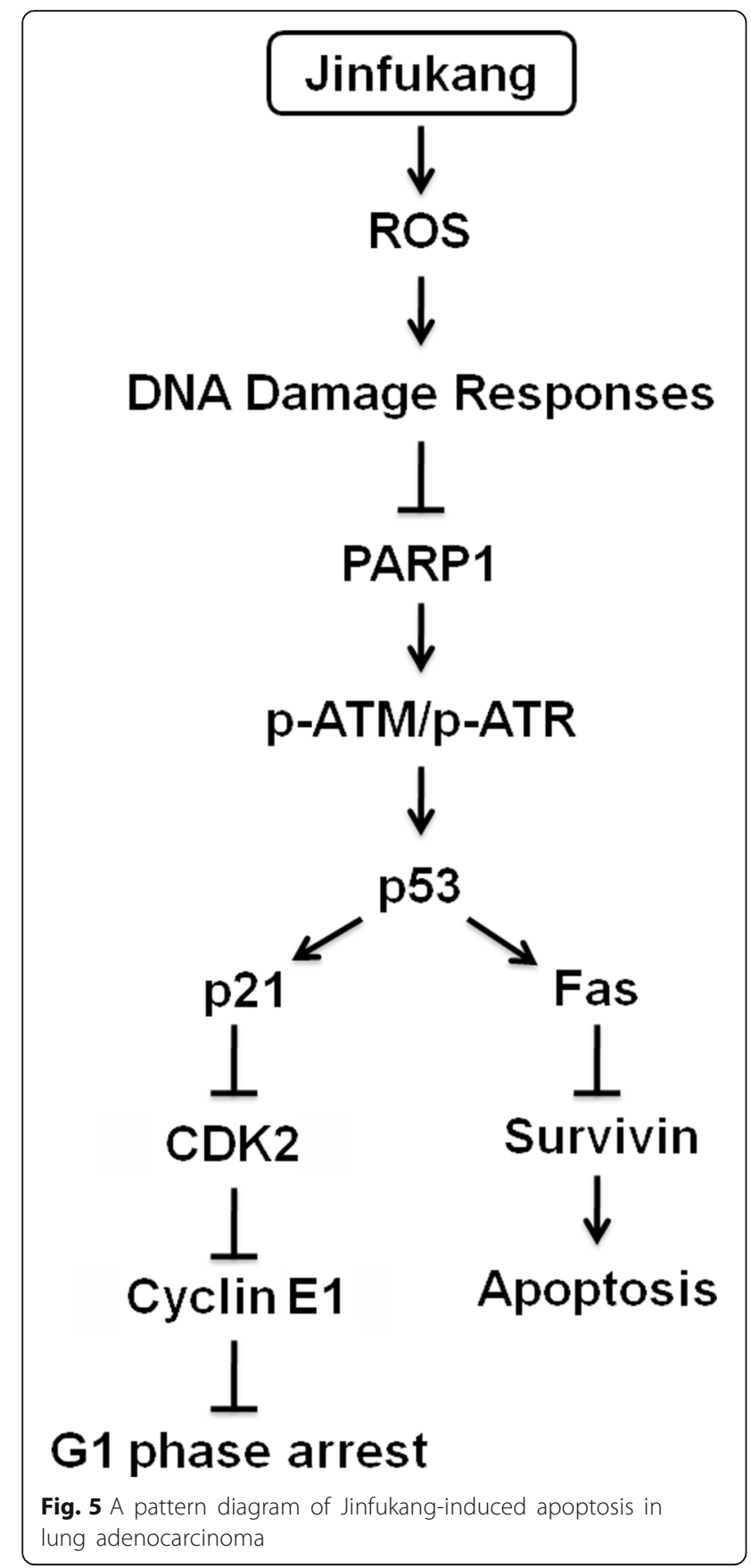

also induce apoptosis of lung adenocarcinoma cells through p53 and MAPK signaling pathways [21, 22]. Nevertheless, it was not found that Jinfukang induced apoptosis in lung cancer cells by intervening ROS pathway. Previous studies have confirmed that increased ROS levels can cause DNA damage and induce cell apoptosis [26]. Hence, we conducted research on this pathway and found that Jinfukang can significantly increase the levels of ROS in CTC-TJH-01 cells. Furthermore, Jinfukang-induced increase in ROS and cell death can partly reversed when cotreatment with NAC. Our study also found that Jinfukang can induce DNA damage in CTC-TJH-01 cells. DNA damage response mainly involves signal pathways such as ATM/ATR-p53, p38/ MAPK-Atf2 and BMP-Smad1 [27]. Our study found that Jinfukang can increase the expression of PARP1, pATM, p-ATR, p53 and p21 after treatment of the CTC$\mathrm{TJH}-01$ cells. Taken together, these results indicate that Jinfukang-induced apoptosis in CTC-TJH-01 cells through the ROS-mediated ATM/ATR-p53 pathway and DNA damage.

\section{Conclusions}

In this study, we have demonstrated that Jinfukang induces cell cycle arrest and ROS-dependent apoptosis in circulating lung cancer cells. Furthermore, we affirmed that Jinfukang induces DNA damage through ROS and the upregulation of p-ATM, p-ATR, p53 and p21. These results provide an insight into the anti-CTC activities of Jinfukang and provide the basis for its clinical application.

\section{Additional files}

Additional file 1: Jinfukang Ingredients. (DOCX $16 \mathrm{~kb}$ )

Additional file 2: Jinfukang fingerprint. (DOC $156 \mathrm{~kb}$ )

\section{Abbreviations}

AJCC: American Joint Committee on Cancer; CTCs: Circulating tumor cells; DCFH-DA: 2,7-Dichlorodihydrofluorescein diacetate; NAC: N-Acetyl-L-cysteine; NSCLC: Non-small cell lung cancer; PI: Propidium iodide; TNM: Tumor node metastasis; TUNEL: TdT-mediated dUTP Nick-End Labeling

\section{Acknowledgments}

This work was supported by the Professor Yu Jin of East China University of Science and Technology (Shanghai, China).

\section{Authors' contributions}

JT and $\mathrm{HL}$ designed the research and $\mathrm{ZQ}, \mathrm{ZZ}$ and $\mathrm{BL}$ performed and wrote the manuscript. CD and YJ helped the analysis of the data. All authors read and approved the final manuscript.

\section{Funding}

The study design, collection, analysis, and interpretation of data and writing the manuscript was supported by the following funding respectively: National Natural Science Foundation of China (81373621, 81774166), Clinical Science and technology innovation project of Shanghai Shenkang Hospital Development Center (SHDC12016114) collection, Excellent Academic Leaders of Health and Family Planning System in Shanghai (2017BR044), Sponsored by Shanghai Sailing Program (17YF1419700), and Longhua Medical Scholar (LYTD-39)

\section{Availability of data and materials}

All data generated or analysed during this study are included in this published article (and its additional information files).

Ethics approval and consent to participate

This study was approved by the Ethics Committee of Longhua hospital, Shanghai University of Traditional Chinese Medicine.

Consent for publication

Not applicable. 


\section{Competing interests}

The authors declare that they have no competing interests.

Received: 26 July 2018 Accepted: 18 July 2019

Published online: 07 August 2019

\section{References}

1. Allemani C, Matsuda T, Di Carlo V, Harewood R, Matz M, Niksic M, Bonaventure A, Valkov M, Johnson CJ, Esteve J, et al. Global surveillance of trends in cancer survival 2000-14 (CONCORD-3): analysis of individual records for 37513025 patients diagnosed with one of 18 cancers from 322 population-based registries in 71 countries. Lancet. 2018:391(10125):1023-75.

2. Antonia SJ, Villegas A, Daniel D, Vicente D, Murakami S, Hui R, Yokoi T, Chiappori A, Lee KH, De WM. Durvalumab after chemoradiotherapy in stage III non-small-cell lung cancer. N Engl J Med. 2017;377(20):1919.

3. Hirsch FR, Scagliotti GV, Mulshine JL, Kwon R, Jr CW, Wu YL, Paz-Ares L. Lung cancer: current therapies and new targeted treatments. Lancet. 2016; 389(10066):299.

4. Wu YL, Cheng Y, Zhou X, Lee KH, Nakagawa K, Niho S, Tsuji F, Linke R, Rosell R, Corral J. Dacomitinib versus gefitinib as first-line treatment for patients with EGFR-mutation-positive non-small-cell lung cancer (ARCHER 1050): a randomised, open-label, phase 3 trial. Lancet Oncol. 2017. https:// doi.org/10.1016/S1470-2045(17)30608-3.

5. Vansteenkiste JF, Cho BC, Vanakesa T, De Pas T, Zielinski M, Kim MS, Jassem J, Yoshimura M, Dahabreh J, Nakayama H, et al. Efficacy of the MAGE-A3 cancer immunotherapeutic as adjuvant therapy in patients with resected MAGE-A3-positive non-small-cell lung cancer (MAGRIT): a randomised, double-blind, placebo-controlled, phase 3 trial. Lancet Oncol. 2016;17(6): 822-35

6. Moslehi JJ, Salem JE, Sosman JA, Lebrun-Vignes B, Johnson DB. Increased reporting of fatal immune checkpoint inhibitor-associated myocarditis. Lancet. 2018:391(10124):933.

7. Weber GF. Why does cancer therapy lack effective anti-metastasis drugs? Cancer Lett. 2013;328(2):207-11.

8. Syrigos K, Fiste O, Charpidou A, Grapsa D. Circulating tumor cells count as a predictor of survival in lung cancer. Crit Rev Oncol Hematol. 2018;125:60-8.

9. Giuliano AE, Edge SB, Hortobagyi GN. Eighth edition of the AJCC cancer staging manual: breast cancer. Ann Surg Oncol. 2018;25(7):1-3.

10. Lee SB, Sohn G, Kim J, Chung IY, Lee JW, Kim HJ, Ko BS, Son BH, Ahn SH. A retrospective prognostic evaluation analysis using the 8th edition of the American joint committee on cancer staging system for breast cancer. Breast Cancer Res Treat. 2018;23(8):1-10.

11. Hashimoto M, Tanaka F, Yoneda K, Takuwa T, Matsumoto S, Okumura Y, Kondo N, Tsujimura T, Nakano T, Hasegawa S. Positive correlation between postoperative tumor recurrence and changes in circulating tumor cell counts in pulmonary venous blood (pvCTC) during surgical manipulation in non-small cell lung cancer. J Thorac Dis. 2018;10(1):298-306.

12. Liu J, Shi Z, Li H, Xu Z, Zhu Y, Zhao L, Hong G, Liu L, Zhu H, Hui Z. Clinical observation on 271 cases of non-small cell lung cancer treated with yifei kangliu yin. 中国结合医学杂志:英文版. 2001;7(4):247-50.

13. Jiao L, Wang $Y$, Ling $X$, Ming Y. Lung cancer prevention and therapy using the JinFuKang herbal mixture. Curr Pharmacol Rep. 2015;1(5):346-53.

14. Wang Z, Wu W, Wang Z, Tang Y, Deng Y, Xu L, Tian J, Shi Q. Ex vivo expansion of circulating lung tumor cells based on one-step microfluidicsbased immunomagnetic isolation. Analyst. 2016;141(12):3621-5.

15. Zheng Y, Tian J. Development of rapid and highly sensitive HSPA1A promoter-driven;luciferase reporter system for assessing oxidative stress associated; with low-dose photodynamic therapy. Cell Stress Chaperones. 2013;18(2):203-13.

16. Jiang ST, Han SY, Pang LN, Jiao YN, He XR, Li PP. Bu-Fei decoction and modified Bu-Fei decoction inhibit the growth of non-small cell lung cancer, possibly via inhibition of apurinic/apyrimidinic endonuclease 1. Int J Mol Med. 2018:41(4):2128-38.

17. Que Z, Zou F, Zhang A, Zheng Y, Bi L, Zhong J, Tian J, Liu J. Ganoderic acid Me induces the apoptosis of competent $T$ cells and increases the proportion of Treg cells through enhancing the expression and activation of indoleamine 2,3-dioxygenase in mouse lewis lung cancer cells. Int Immunopharmacol. 2014;23(1):192-204.

18. Liu Y, Cao X. Characteristics and significance of the pre-metastatic niche. Cancer Cell. 2016;30(5):668-81.
19. Que Z, Luo B, Zhou Z, Dong C, Jiang Y, Wang L, Shi Q, Tian J. Establishment and characterization of a patient-derived circulating lung tumor cell line in vitro and in vivo; 2019.

20. Cassileth BR, Rizvi N, Deng G, Yeung KS, Vickers A, Guillen S, Woo D, Coleton M, Kris MG. Safety and pharmacokinetic trial of docetaxel plus an Astragalus -based herbal formula for non-small cell lung cancer patients. Cancer Chemother Pharmacol. 2009;65(1):67-71.

21. Lu J, Chen J, Xu N, Wu J, Kang Y, Shen T, Kong H, Ma C, Cheng M, Shao Z. Activation of AIFM2 enhances apoptosis of human lung cancer cells undergoing toxicological stress. Toxicol Lett. 2016;258:227.

22. Lu J, Zhang X, Shen T, Ma C, WU J, Kong H, Tian J, Shao Z, Zhao X, Xu L. Epigenetic profiling of $\mathrm{H} 3 \mathrm{~K} 4 \mathrm{Me} 3$ reveals herbal medicine Jinfukang-Induced epigenetic alteration is involved in anti-lung cancer activity. Evid Based Complement Alternat Med. 2016;2016(4):7276161.

23. He HL, Dan W, Jie T, Zhou XM, Li JX, Ling X. Jin Fu Kang oral liquid inhibits lymphatic endothelial cells formation and migration. Evid Based Complement Alternat Med. 2016;2016(3):1-10.

24. Zhou XM, Wang D, He HL, Tang J, Wu J, Xu L, Li JX. Bone marrow derived mesenchymal stem cells involve in the lymphangiogenesis of lung cancer and Jinfukang inhibits the involvement in vivo. J Cancer. 2017;8(10):1786-94.

25. Sun Q, Qing W, Qi R, Zou M, Gong L, Liu Y, Li DW. Inhibition of Sumoylation alleviates oxidative stress-induced retinal pigment epithelial cell senescence and represses proinflammatory gene expression. Curr Mol Med. 2018;18(9): $575-83$.

26. Tse AK, Chen YJ, Fu XQ, Su T, Li T, Guo H, Zhu PL, Kwan HY, Cheng BC, Cao $H H$, et al. Sensitization of melanoma cells to alkylating agent-induced DNA damage and cell death via orchestrating oxidative stress and IKKbeta inhibition. Redox Biol. 2017;11:562-76.

27. Mcnally JP, Millen SH, Chaturvedi V, Lakes N, Terrell CE, Elfers EE, Carroll KR, Hogan SP, Andreassen PR, Kanter J. Manipulating DNA damage-response signaling for the treatment of immune-mediated diseases. Proc Natl Acad Sci U S A. 2017;114(24):201703683.

\section{Publisher's Note}

Springer Nature remains neutral with regard to jurisdictional claims in published maps and institutional affiliations.
Ready to submit your research? Choose BMC and benefit from:

- fast, convenient online submission

- thorough peer review by experienced researchers in your field

- rapid publication on acceptance

- support for research data, including large and complex data types

- gold Open Access which fosters wider collaboration and increased citations

- maximum visibility for your research: over $100 \mathrm{M}$ website views per year

At $\mathrm{BMC}$, research is always in progress.

Learn more biomedcentral.com/submissions 